\title{
Availability of a Periodically Inspected System Maintained through Several Minimal Repairs before a Replacement or a Perfect Repair
}

\author{
Jun Yang, Tingting Gang, and Yu Zhao \\ School of Reliability and Systems Engineering, Beihang University, Beijing 100191, China \\ Correspondence should be addressed to Jun Yang; 08264@buaa.edu.cn
}

Received 13 April 2013; Accepted 2 June 2013

Academic Editor: Luca Guerrini

Copyright (C) 2013 Jun Yang et al. This is an open access article distributed under the Creative Commons Attribution License, which permits unrestricted use, distribution, and reproduction in any medium, provided the original work is properly cited.

\begin{abstract}
The instantaneous availability of a periodically inspected system with several minimal repairs before a replacement or a perfect repair is studied. First, we investigate two concrete minimal repair models with periodic inspection and constant repair time and give their instantaneous availability formulas. Then the instantaneous availability of the proposed model with constant repair times is presented by a set of recursive formulas, and its piecewise monotonicity is also shown. An example is presented to illustrate its application.
\end{abstract}

\section{Introduction}

A system is either in the upstate or in the downstate at any instant $t$, where the term "up-state" means that the system still works and the term "down-state" means that the system does not work. The state of the system at instant $t$ can be written as,

$$
X_{t}=\left\{\begin{array}{l}
1, \\
\text { the system is in the upstate at instant } t, \\
0, \\
\text { the system is in the downstate at instant } t
\end{array}\right.
$$

and the instantaneous availability at instant $t$ can be defined as

$$
A(t)=P\left(X_{t}=1\right) .
$$

The purpose of repair is to make the failed system work well. From the viewpoint of reliability, replacement or perfect repair may be the first choice, where perfect repair means the failed system is repaired to as good as new in [1]. The availability of the system under periodic inspection with different perfect repair or replacement policy has been studied in [2,3]. Cui et al. [4] presented an availability model for storage products under periodic inspection, and Cui et al. [5] analyzed the single-unit repairable system and gave formulas for reliability indexes by state aggregations. However, from the viewpoint of engineering or economics, replacement or perfect repair is not always desirable, since they may be too expensive or need too much time. Hence, imperfect repair policy is often adopted in practice. The imperfect repair model proposed in [6] has been studied extensively [7-14]. In the imperfect repair model, if the system fails, it will return to the good-as-new state by perfect repair with probability $p$, and it will return to a functioning state as bad as old by minimal repair with probability $q=$ $1-p$. Since the probability $p$ is hard to obtain, the imperfect model is not widely used in practice, and another promise model is proposed in [15], where the system is maintained by several imperfect repairs before a replacement or a perfect repair. Notice that periodic inspection is often adopted in practice, and we focus on the periodically inspected system maintained through several minimal repairs before a replacement or a perfect repair. The structure sketch of the above system can be seen in Figure 1, and some basic assumptions are presented in detail as follows:

(1) at instant $t=0$, the system is assumed to be normal, and from then on it is inspected regularly at time instants $\tau_{1}=\tau, \tau_{2}=2 \tau, \ldots$; 


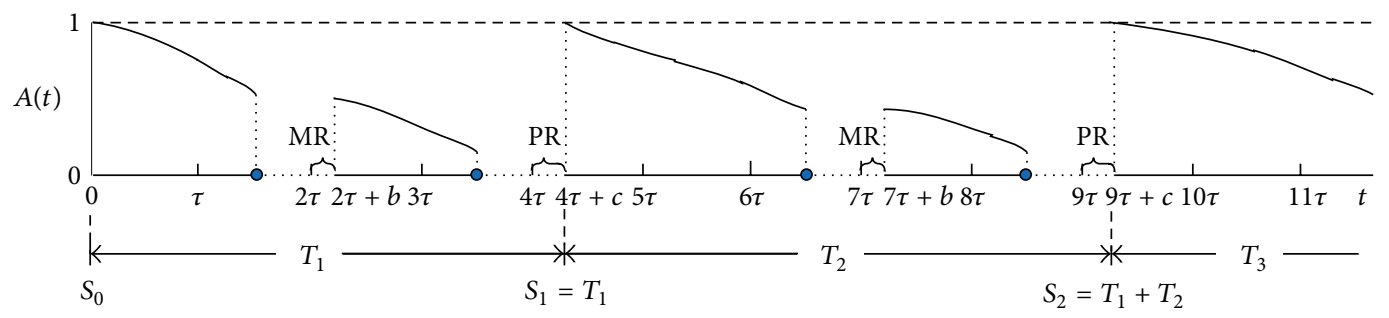

FIGURE 1: The structure sketch of a periodically inspected system maintained through several minimal repairs before a replacement or a perfect repair, where "•" denotes the system failure, MR and PR denote minimal repair and perfect repair or replacement, respectively, and $m$ is prefixed as 2 .

(2) for each inspection, if the system is functioning, it will continue its work without any repair; if the system fails, the failure times $k$ will be recorded at first, and the prefixed minimal repair times $m-1$ between two consecutive replacements or perfect repairs can be determined from the constraints of cost, resource, and maintainability in advance;

(3) if $k \bmod m \neq 0$, the system will be repaired to as bad as old by minimal repair with constant repair time $b(0 \leq$ $b<\tau)$; if $k \bmod m=0$, the system will be replaced by a good new one or repaired to the good-as-new state by perfect repair with constant repair time $c(0 \leq c<$ $\tau)$. The system will immediately work after repair or replacement;

(4) each replacement or the completion of each perfect repair can be regarded as a renewal.

The time interval between two consecutive renewals is denoted by $T_{i}(i=1,2, \ldots)$, and let

$$
S_{0}=0, \quad S_{j}=\sum_{i=1}^{j} T_{i} ;
$$

that is, $S_{1}=T_{1}$ is the time of the first renewal; $S_{2}=$ $T_{1}+T_{2}$ is the time of the second renewal. In general, $S_{j}$ denotes the time of the $j$ th renewal (see Figure 1). From the previous assumptions, $T_{i}(i=1,2, \ldots)$ are independent, and $T_{i}(i=2,3, \ldots)$ are independent and identically distributed; thus, $\left\{T_{i}, i=1,2, \ldots\right\}$ forms a delayed renewal process. For convenience, the renewal times in time interval $[0, t)$ are denoted by $N_{D}(t)$, and the last renewal time is denoted by $S_{N_{D}(t)}$.

The instantaneous availability $A(t)$ is focused on under the following two models:

(A) $m-1$ minimal repairs are allowed before replacement with a new one;

(B) $m-1$ minimal repairs are allowed before a perfect repair.

For convenience, let $b_{k}=\tau * k+b, c_{k}=\tau *$ $k+c$, where $k=1,2, \ldots$. For model $\mathrm{B}$, distributions of $T_{1}$ and $T_{i}(i=2,3, \ldots)$ are denoted as $G(t)$ and $H(t)$, respectively, and for model $\mathrm{A}, T_{1}, T_{2}, \ldots$ are independent and identically distributed, and $G(t)=H(t)$. From the viewpoint of rsseliability, the effect of replacement and perfect repair is identical; in other words, replacement can be considered as a special perfect repair whose repair time is 0 . Hence, model A can be considered as a special case of model B in mathematics, and model B is studied in detail in this paper. If $m=+\infty$, model $\mathrm{B}$ degenerates into the ordinary minimal repair model with periodic inspection and constant repair time, which is discussed in Section 2; if $m=1$, model B degenerates into the perfect repair model with periodic inspection and constant repair time $[2,3]$.

The instantaneous availability of the ordinary minimal repair model with periodic inspection and constant repair time is presented in Section 2; in Section 3, the instantaneous availability of the system under model B is given, and its monotonicity is also discussed; and some conclusions are given in the end.

\section{System under Periodic Inspections with Minimal Repair}

In order to study the instantaneous availability of the proposed model, a minimal repair model with periodic inspection and constant repair time is discussed first. For this minimal repair model, the system starts work at instant 0 and will be inspected at regular time instants $\tau_{k}=k * \tau$, $k=1,2, \ldots$. At instant $\tau_{k}$, if the system fails, it will be repaired to as bad as old by minimal repair with constant repair time $b(0 \leq b \leq \tau)$; otherwise, it will continue its work without any repair. For convenience, this model is referred to as minimal repair model $I$.

Assume that the distribution of time to first failure of the system is $F(t)$ and its reliability $R(t)=1-F(t)$, and the system instantaneous availability under minimal repair model $I$ is denoted by $A_{1}(t)$. In [16], the following theorem and corollary are given.

Theorem 1. For minimal repair model I, the instantaneous availability of the system is

$$
A_{1}(t)=\left\{\begin{aligned}
& R(t) \\
& 0 \leq t \leq \tau_{1}, \\
& \frac{A_{1}\left(\tau_{k}\right)}{R\left(\tau_{k}\right)}, \\
& \tau_{k}<t \leq b_{k}, k=1,2, \ldots, \\
& R(t) \times\left[\frac{A_{1}\left(\tau_{k}\right)}{R\left(\tau_{k}\right)}+\frac{1-A_{1}\left(\tau_{k}\right)}{R\left(b_{k}\right)}\right], \\
& b_{k}<t \leq \tau_{k+1}, k=1,2, \ldots
\end{aligned}\right.
$$


Corollary 2. For minimal repair model I, the availability of the system is piecewise monotonic decreasing, and it arrives at its minimum values at instant $b_{k}, k=1,2, \ldots$.

The assumption of minimal repair model II is similar to that of minimal repair model I, except that the system starts work at instant $(0 \leq d<\tau)$. The system instantaneous availability under minimal repair model II is denoted by $A_{2}(t)$. Similarly, $A_{2}(t)$ is expressed as follows:

$$
A_{2}(t)=\left\{\begin{aligned}
R(t-d), \\
d \leq t \leq \tau_{1}, \\
R(t-d) \frac{A_{2}\left(\tau_{k}\right)}{R\left(\tau_{k}-d\right)}, \\
\tau_{k}<t \leq b_{k}, k=1,2, \ldots, \\
R(t-d)\left\{\frac{A_{2}\left(\tau_{k}\right)}{R\left(\tau_{k}-d\right)}+\frac{\left[1-A_{2}\left(\tau_{k}\right)\right]}{R\left(b_{k}-d\right)}\right\}, \\
b_{k}<t \leq \tau_{k+1}, k=1,2, \ldots
\end{aligned}\right.
$$

And its instantaneous availability is also piecewise monotonic decreasing, and it arrives at its minimum values at instant $b_{k}$, $k=1,2, \ldots$.

\section{Availability of a Periodically Inspected System Maintained by $m-1$ Minimal Repairs before a Perfect Repair}

In this section, the instantaneous availability for model B is discussed. Since there is no perfect repair in time interval $\left[0, c_{m}\right]$ from the assumptions of model $\mathrm{B}$, then the system instantaneous availability of model B is the same as that of minimal repair model $I$, and we have the following result.

Proposition 3. For model B,

$$
A(t)=A_{1}(t), \quad t \in\left[0, c_{m}\right]
$$

Since model $B$ under condition $T_{1}>t$ and condition $T_{2}>$ $t-c$ is equal to minimal repair model $I$ and minimal repair model II with $d=c$, respectively, one has

$$
\begin{gathered}
P\left\{X_{t}=1 \mid T_{1}>t\right\}=A_{1}(t), \\
P\left\{X_{t}=1 \mid T_{2}>t-c\right\}=A_{2}(t) .
\end{gathered}
$$

Proposition 4. For model B,

$$
\begin{aligned}
A(t)=\sum_{k=m}^{n} \sum_{j=1}^{[k / m]} P & \left\{S_{j}=c_{k}\right\} A_{2}\left(t-\tau_{k}\right) P\left\{T_{2}>t-c_{k}\right\} \\
& +A_{1}(t) P\left\{T_{1}>t\right\},
\end{aligned}
$$

where $t \in\left[c_{n}, c_{n+1}\right], n=m, m+1, \ldots,[x]$ denotes the largest integer not exceeding $x$, and $S_{j}$ is the time of the jth renewal.
Proof. On $[0, t)$, the time of the last renewal $S_{j}$ will be $c_{k}, k=$ $m, m+1, \ldots, n$; then the system will be renewed at most $[k / m]$ times. Hence, by the total probability theorem, we have

$$
\begin{aligned}
A(t)= & P\left\{X_{t}=1\right\} \\
= & P\left\{X_{t}=1, T_{1}>t\right\} \\
& +\sum_{k=m}^{n} \sum_{j=1}^{[k / m]} P\left\{X_{t}=1, S_{j}=c_{k}, T_{j+1}>t-c_{k}\right\} \\
= & P\left\{X_{t}=1 \mid T_{1}>t\right\} P\left\{T_{1}>t\right\} \\
& +\sum_{k=m}^{n} \sum_{j=1}^{[k / m]} P\left\{S_{j}=c_{k}\right\} P\left\{X_{t}=1, T_{j+1}>t-c_{k}\right\} \\
= & A_{1}(t) P\left\{T_{1}>t\right\} \\
& +\sum_{k=m}^{n} \sum_{j=1}^{[k / m]} P\left\{S_{j}=c_{k}\right\} P\left\{X_{t}=1, T_{2}>t-c_{k}\right\} \\
= & A_{1}(t) P\left\{T_{1}>t\right\} \\
& +\sum_{k=m}^{n} \sum_{j=1}^{[k / m]} P\left\{S_{j}=c_{k}\right\} A_{2}\left(t-\tau_{k}\right) \\
& \times P\left\{T_{2}>t-c_{k}\right\},
\end{aligned}
$$

where the three but last equations follow from the fact that $T_{i}, i=1,2, \ldots$ are independent and the two but last equations follow from the fact that $T_{i}, i=2,3, \ldots$ are independent and identically distributed. Thus, the proof is completed.

For convenience, let $g_{k}=P\left\{T_{1}=c_{k}\right\}, k=m, m+1, \ldots$ For minimal repair model $I$, the event that there are $j(k \geq j \geq$ 1) failures in $\left[0, \tau_{k}\right]$ and one failure in $\left(\tau_{k-1}, \tau_{k}\right]$ is denoted by $B(k, j, 1)$; and the event that there are $j(k \geq j \geq 1)$ failures in $\left[0, \tau_{k}\right]$ and no failure in $\left(\tau_{k-1}, \tau_{k}\right]$ is denoted by $B(k, j, 0)$. The probabilities of $B(k, j, 0), j=0,1, \ldots, k-1$ and $B(k, j, 1), j=1,2, \ldots, k$ can be obtained by the argument of mathematical induction

$$
\begin{gathered}
P\{B(k, 0,0)\}=R\left(\tau_{k}\right), \\
P\{B(k, 1,1)\}=R\left(\tau_{k-1}\right)-R\left(\tau_{k}\right), \\
P\{B(k, k, 1)\}=\left[R\left(b_{k-1}\right)-R\left(\tau_{k}\right)\right] \\
\times P\{B(k-1, k-1,1)\}, \\
P\{B(k, j, 1)\}=\left[R\left(b_{k-1}\right)-R\left(\tau_{k}\right)\right] \\
\times P\{B(k-1, j-1,1)\} \\
+\left[R\left(\tau_{k-1}\right)-R\left(\tau_{k}\right)\right] \\
\times P\{B(k-1, j-1,0)\},
\end{gathered}
$$




$$
\begin{aligned}
P\{B(k, k-1,0)\}= & {\left[1-R\left(b_{k-1}\right)+R\left(\tau_{k}\right)\right] } \\
& \times P\{B(k-1, k-1,1)\}, \\
P\{B(k, j, 0)\}= & {\left[1-R\left(b_{k-1}\right)+R\left(\tau_{k}\right)\right] } \\
& \times P\{B(k-1, j, 1)\} \\
& +\left[1-R\left(\tau_{k-1}\right)+R\left(\tau_{k}\right)\right] \\
& \times P\{B(k-1, j, 0)\},
\end{aligned}
$$

where $1<j \leq k-1$.

From the definition of $B(k, j, 1)$ and model $\mathrm{B}$, we have $\left\{T_{1}=c_{k}\right\}=B(k, m, 1)$; then

$$
g_{k}=P\left\{T_{1}=c_{k}\right\}=P\{B(k, m, 1)\}, \quad k=m, m+1, \ldots,
$$

and they can be calculated by (10).

For convenience, let $h_{k}=P\left\{T_{2}=\tau_{k}\right\}, k=m, m+1, \ldots$ For minimal repair model II with $d=c$, the event that there are $j(k \geq j \geq 1)$ failures in $\left[c, \tau_{k}\right]$ and one failure in $\left(\tau_{k-1}, \tau_{k}\right]$ is denoted by $D(k, j, 1)$; and the event that there are $j(k \geq j \geq$ 1) failures in $\left[c, \tau_{k}\right]$ and no failure in $\left(\tau_{k-1}, \tau_{k}\right]$ is denoted by $D(k, j, 0)$. The recursive formula of probabilities of $D(k, j, 0)$, $j=0,1, \ldots, k-1$ and $D(k, j, 1), j=1,2, \ldots, k-1$ can be obtained by the argument of mathematical induction

$$
\begin{aligned}
& P\{D(1,1,1)\}=1-R(\tau-c), \\
& P\{D(k, 0,0)\}=R\left(\tau_{k}-c\right), \\
& P\{D(k, 1,1)\}=R\left(\tau_{k-1}-c\right)-R\left(\tau_{k}-c\right), \\
& P\{D(k, k, 1)\}=\left[R\left(b_{k-1}-c\right)-R\left(\tau_{k}-c\right)\right] \\
& \times P\{D(k-1, k-1,1)\}, \\
& P\{D(k, j, 1)\}=\left[R\left(b_{k-1}-c\right)-R\left(\tau_{k}-c\right)\right] \\
& \times P\{D(k-1, j-1,1)\} \\
& +\left[R\left(\tau_{k-1}-c\right)-R\left(\tau_{k}-c\right)\right] \\
& \times P\{D(k-1, j-1,0)\}, \\
& 1<j \leq k-1 \text {, } \\
& P\{D(k, k-1,0)\}=\left[1-R\left(b_{k-1}-c\right)+R\left(\tau_{k}-c\right)\right] \\
& \times P\{D(k-1, k-1,1)\}, \\
& P\{D(k, j, 0)\}=\left[1-R\left(b_{k-1}-c\right)+R\left(\tau_{k}-c\right)\right] \\
& \times P\{D(k-1, j, 1)\} \\
& +\left[1-R\left(\tau_{k-1}-c\right)+R\left(\tau_{k}-c\right)\right] \\
& \times P\{D(k-1, j, 0)\}, \\
& 1<j \leq k-1 \text {. }
\end{aligned}
$$

From the definition of $D(k, j, 1)$ and model $\mathrm{B}$, we have $\left\{T_{2}=\tau_{k}\right\}=D(k, m, 1)$; then

$$
h_{k}=P\left\{T_{2}=\tau_{k}\right\}=P\{D(k, m, 1)\}, \quad k=m, m+1, \ldots,
$$

and they can be calculated by (12).

For the first renewal time $T_{1}$, let $Q_{1}(t)=P\left\{T_{1}>t\right\}$. In this case, the system starts work at instant 0 , and from (11), we have

$$
\begin{aligned}
P\left\{T_{1}>t\right\} & =\sum_{k=[(t-c) / \tau]+1}^{\infty} P\left\{T_{1}=c_{k}\right\} \\
& =1-\sum_{k=m}^{[(t-c) / \tau]} P\left\{T_{1}=c_{k}\right\} \\
& =1-\sum_{k=m}^{[(t-c) / \tau]} g_{k} ;
\end{aligned}
$$

then

$$
Q_{1}(t)=1-\sum_{k=m}^{[(t-c) / \tau]} g_{k}
$$

For the second renewal time, let $Q_{2}(t)=P\left(T_{2}>t\right)$. In this case, the system starts work at instant $c$, and from (13), we have

$$
\begin{aligned}
P\left\{T_{2}>t\right\} & =\sum_{k=[t / \tau]+1}^{\infty} P\left\{T_{2}=\tau_{k}\right\} \\
& =1-\sum_{k=m}^{[t / \tau]} P\left\{T_{2}=\tau_{k}\right\} \\
& =1-\sum_{k=m}^{[t / \tau]} h_{k} ;
\end{aligned}
$$

then

$$
Q_{2}(t)=1-\sum_{k=m}^{[t / \tau]} h_{k}
$$

From the fact that $T_{1}$ and $T_{i}(i=2,3, \ldots)$ follow distributions $G$ and $H$, respectively, it is easy to know that the time of the $j$ th renewal $S_{j}=\sum_{k=1}^{j} T_{k}$ follows distribution $G * H_{j-1}$, where $*$ means the convolution of distribution functions, and the value set of $S_{j}$ is $\left\{c_{k}: k=m j, m j+1, \ldots\right\}$. For convenience, probability generating functions of distributions $G$ and $H$ are denoted by $g(s)$ and $h(s)$, respectively, which can be expressed as $g(s)=\sum_{l=m}^{\infty} g_{l} s^{l}$ and $h(s)=\sum_{i=m}^{\infty} h_{i} s^{i}$, respectively.

Let $P\left\{S_{j}=c_{k}\right\}=v_{k}^{(j)}$ and $G * H_{j-1}=\left\{v_{k}^{(j)}: k=m j, m j+\right.$ $1, \ldots\}$, so the probability generating function of $G * H_{j-1}$ can be expressed as $\sum_{k=m j}^{\infty} v_{k}^{(j)} s^{k}$. Since $T_{1}, T_{2}, \ldots$ forms a delay 
renewal process and consequently $S_{j}(j=1,2, \ldots)$ forms a delay renewal process, then

$$
\sum_{k=m j}^{\infty} v_{k}^{(j)} s^{k}=\left(\sum_{l=m}^{\infty} g_{l} s^{l}\right) \times\left(\sum_{i=m}^{\infty} h_{i} s^{i}\right)^{j-1} .
$$

Through comparison of similar items between two sides of (18), we have

$$
\begin{gathered}
v_{k}^{(1)}=g_{k}, \quad k=m, m+1, \ldots \\
v_{k}^{(2)}=\sum_{i=m}^{k-m} v_{i}^{(1)} h_{k-i}, \quad k=2 m, 2 m+1, \ldots \\
\vdots \\
v_{k}^{(j)}=\sum_{i=m(j-1)}^{k-m} v_{i}^{(j-1)} h_{k-i}, \quad k=m j, m j+1, \ldots
\end{gathered}
$$

From the previous results, we have the following theorem and corollary.

Theorem 5. The instantaneous availability of the system under model $B$ is

$$
A(t)=\left\{\begin{array}{l}
A_{1}(t), \\
0 \leq t \leq c_{m}, \\
A_{1}(t) Q_{1}(t)+\sum_{k=m}^{n} \sum_{j=1}^{[k / m]} v_{k}^{(j)} A_{2}\left(t-\tau_{k}\right) Q_{2}\left(t-c_{k}\right), \\
c_{n} \leq t<c_{n+1}, n=m, m+1, \ldots
\end{array}\right.
$$

Corollary 6. The instantaneous availability of the system under model $B$ is piecewise monotonic decreasing, and it arrives at its minimum values at instant $b_{i}, i=1,2, \ldots$.

Proof. On $\left[0, c_{m}\right), A(t)=A_{1}(t)$, and from Corollary 2, we have that $A(t)$ is piecewise monotonic decreasing and arrives at its minimal values at instant $b_{i}, i=1,2, \ldots, m$.

On $\left[c_{n}, c_{n+1}\right), n=m, m+1, \ldots$, from (15) and (17), $Q_{1}(t)=$ $1-\sum_{\mathrm{l}=m}^{n} g_{l}$ and $Q_{2}\left(t-c_{k}\right)=1-\sum_{l=m}^{n-k} h_{l}$; that is, $Q_{1}(t)$ and $Q_{2}\left(t-c_{k}\right)$ are fixed constants on $\left[c_{n}, c_{n+1}\right)$. Similarly, it can be proved that $v_{k}^{(j)}$ is also a fixed constant. From Theorem 5 , $A(t)=A_{1}(t) Q_{1}(t)+\sum_{k=m}^{n} \sum_{j=1}^{[k / m]} v_{k}^{(j)} A_{2}\left(t-\tau_{k}\right) Q_{2}\left(t-c_{k}\right)$, and from Corollary 2, $A_{1}(t)$ and $A_{2}\left(t-\tau_{k}\right)$ are piecewise monotonic decreasing and arrive at their minimum values at instant $b_{i}, i=1,2, \ldots$; then $A(t)$ is piecewise monotonic decreasing and arrives at its minimum values at instant $b_{i}$, $i=1,2, \ldots$. Thus, the proof is completed.

For model A, the periodically inspected system is maintained by $m-1$ minimal repairs before replacement. As mentioned previously, model $\mathrm{A}$ is equal to a special case of model $\mathrm{B}$ whose perfect repair time is 0 . Hence, the $c$ in (20) is replaced by 0 , and the instantaneous availability of model A can be given. And notice that $T_{i}, i=1,2, \ldots$ form

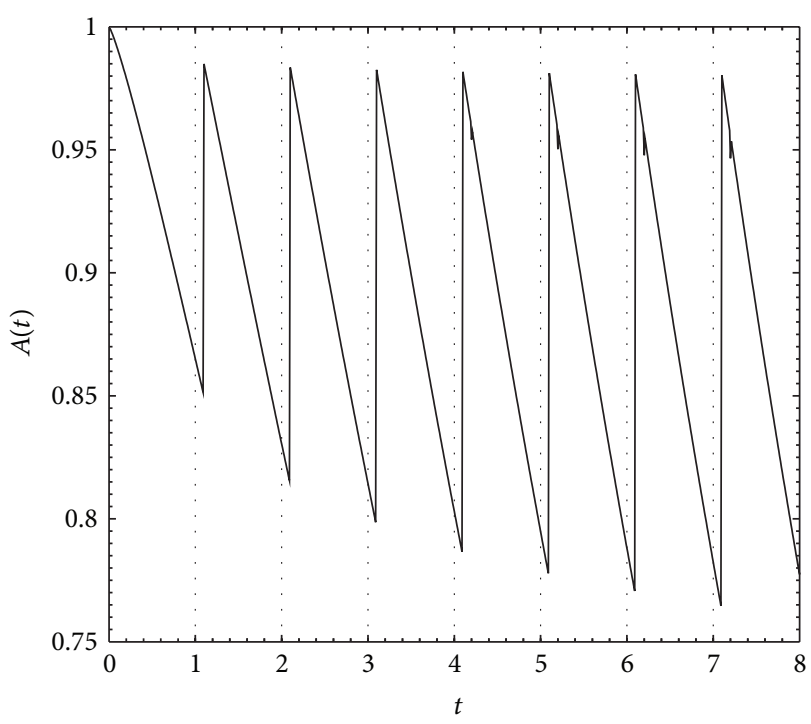

FIGURE 2: The availability curve under model B with $\tau=1.0, b=0.1$, $c=0.2$, and $m=3$.

a renewal process under model $\mathrm{A}$ and they are independent and identically distributed; thus, $A_{2}(t)=A_{1}(t)$ and $Q_{2}(t)=$ $Q_{1}(t)$ under model A. Hence, we have the following theorem and corollary.

Theorem 7. The instantaneous availability of the system under model $A$ is

$$
A(t)=\left\{\begin{array}{l}
A_{1}(t), \\
0 \leq t \leq \tau_{m}, \\
A_{1}(t) Q_{1}(t)+\sum_{k=m}^{n} \sum_{j=1}^{[k / m]} v_{k}^{(j)} A_{1}\left(t-\tau_{k}\right) Q_{1}\left(t-c_{k}\right), \\
\tau_{n} \leq t<\tau_{n+1}, n=m, m+1, \ldots
\end{array}\right.
$$

Corollary 8. The instantaneous availability of the system under model $A$ is piecewise monotonic decreasing, and it arrives at its minimum values at instant $b_{i}, i=1,2, \ldots$.

Example 9. Suppose the distribution of time to first failure of a system is $F(t)=1-\exp \left\{-(t / 5)^{1.2}\right\}$, and it is periodically inspected and maintained by 2 minimal repairs before a perfect repair, where the regular inspection interval $\tau=1.0$ and the constant repair times of minimal repair and perfect repair are 0.1 and 0.2 , respectively. In other words, it is under model B with $\tau=1.0, b=0.1, c=0.2$, and $m=3$. From Theorem 5, its instantaneous availability $A(t)$ can be calculated, and its availability curve can be seen in Figure 2.

For the previous example, if the perfect repair is replaced by replacement, that is to say, the system is under model $\mathrm{A}$ with $\tau=1.0, b=0.1$, and $m=3$. According to Theorem 7 , its instantaneous availability $A(t)$ can be calculated, and its availability curve can be seen in Figure 3.

The availability curves in Figures 2 and 3 show that the instantaneous availability of the periodically inspected 


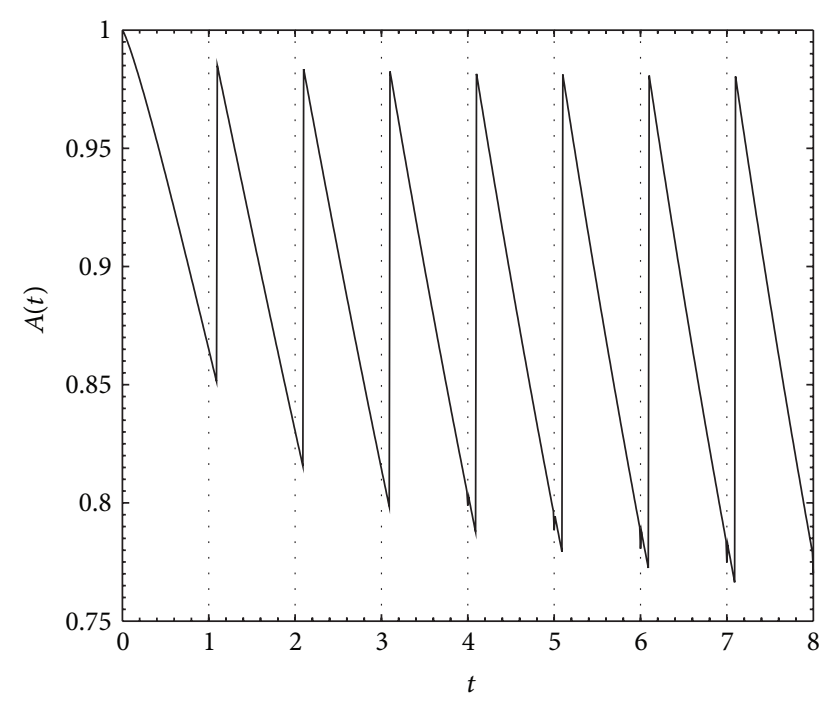

FIgURE 3: The availability curve under model A with $\tau=1.0, b=0.1$, and $m=3$.

system maintained through several minimal repairs before a replacement or a perfect repair is piecewise monotonic decreasing and arrives at its minimum values at instant $b_{k}$, which provides important information for management and decisions of reliability and maintenance. For example, if the instantaneous availability requirement is considered, we can only focus on the instantaneous availability at instant $b_{k}, k=$ $1,2, \ldots$

\section{Conclusion}

The availability of a periodically inspected system maintained through several minimal repairs before a replacement or a perfect repair is studied in this paper. The instantaneous availability of the proposed model is derived by a set of recursive formulas, and it is found that its availability is piecewise monotonic decreasing, which provides theoretical basis for management and decisions in terms of system reliability and maintenance in practice. In particular, the monotonicity of the instantaneous availability can be utilized to optimize the practical reliability work and make our attention focused on some critical time instants.

For simplicity, minimal repair and perfect repair are supposed to take different constant times in this contribution, and this assumption reflects the difference between minimal repair and perfect repair in a certain degree. Availability with random repair time and more realistic assumptions will be investigated in our future research.

\section{Acknowledgments}

This work was supported in part by China NSFC under Grant no. 11001005 and the Fundamental Research Funds for the Central Universities under Grant no. YWF-13-ZY-169.

\section{References}

[1] R. E. Barlow and F. Proschan, Statistical Theory of Reliability and Life Testing, Holt, Rinehart and Winston, New York, NY, USA, 1975.

[2] J. Sarkar and S. Sarkar, "Availability of a periodically inspected system under perfect repair," Journal of Statistical Planning and Inference, vol. 91, no. 1, pp. 77-90, 2000.

[3] L. Cui and M. Xie, "Availability of a periodically inspected system with random repair or replacement times," Journal of Statistical Planning and Inference, vol. 131, no. 1, pp. 89-100, 2005.

[4] L. Cui, X. Zhao, J. Shen, and Y. Xu, "An availability model for storage products under periodical inspections," International Journal of Reliability, Quality and Safety Engineering, vol. 17, no. 2, pp. 89-103, 2010.

[5] L. Cui, S. Du, and A. G. Hawkes, "A study on a single-unit repairable system with state aggregations," IIE Transactions, vol. 44, pp. 1022-1032, 2012.

[6] M. Brown and F. Proschan, "Imperfect repair," Journal of Applied Probability, vol. 20, no. 4, pp. 851-859, 1983.

[7] J.-L. Bon and E. Păltănea, "Convergence of the number of failed components in a Markov system with nonidentical components," Journal of Applied Probability, vol. 38, no. 4, pp. 882-897, 2001.

[8] L. Cui and M. Xie, "Availability analysis of periodically inspected systems with random walk model," Journal of Applied Probability, vol. 38, no. 4, pp. 860-871, 2001.

[9] S. Bloch-Mercier, "Monotone Markov processes with respect to the reversed hazard rate ordering: an application to reliability," Journal of Applied Probability, vol. 38, no. 1, pp. 195-208, 2001.

[10] P. C. Kiessler, G.-A. Klutke, and Y. Yang, "Availability of periodically inspected systems subject to Markovian degradation," Journal of Applied Probability, vol. 39, no. 4, pp. 700-711, 2002.

[11] J. P. Kharoufeh, D. E. Finkelstein, and D. G. Mixon, "Availability of periodically inspected systems with Markovian wear and shocks," Journal of Applied Probability, vol. 43, no. 2, pp. 303317, 2006.

[12] H. Wang and H. Pham, "Availability and maintenance of series systems subject to imperfect repair and correlated failure and repair," European Journal of Operational Research, vol. 174, no. 3, pp. 1706-1722, 2006.

[13] E. Frostig and M. Kenzin, "Availability of inspected systems subject to shocks-a matrix algorithmic approach," European Journal of Operational Research, vol. 193, no. 1, pp. 168-183, 2009.

[14] N. Ebrahimi, "The mean function of a repairable system that is subjected to an imperfect repair policy," IIE Transactions, vol. 41, no. 1, pp. 57-64, 2009.

[15] A. Biswas and J. Sarkar, "Availability of a system maintained through several imperfect repairs before a replacement or a perfect repair," Statistics \& Probability Letters, vol. 50, no. 2, pp. 105-114, 2000.

[16] D. Yu, X. Yan, and G. Y. Li, "The fiducial inference for repaired as old Weibull distributed systems," Chinese Journal of Applied Probability and Statistics, vol. 20, no. 2, pp. 197-206, 2004. 


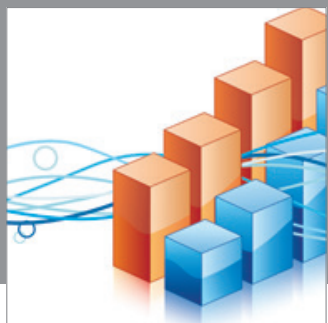

Advances in

Operations Research

mansans

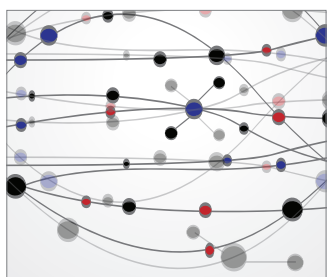

The Scientific World Journal
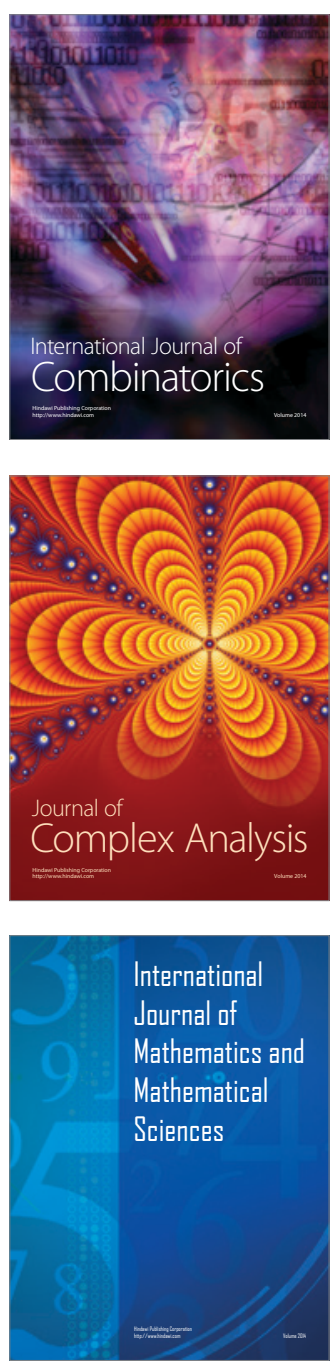
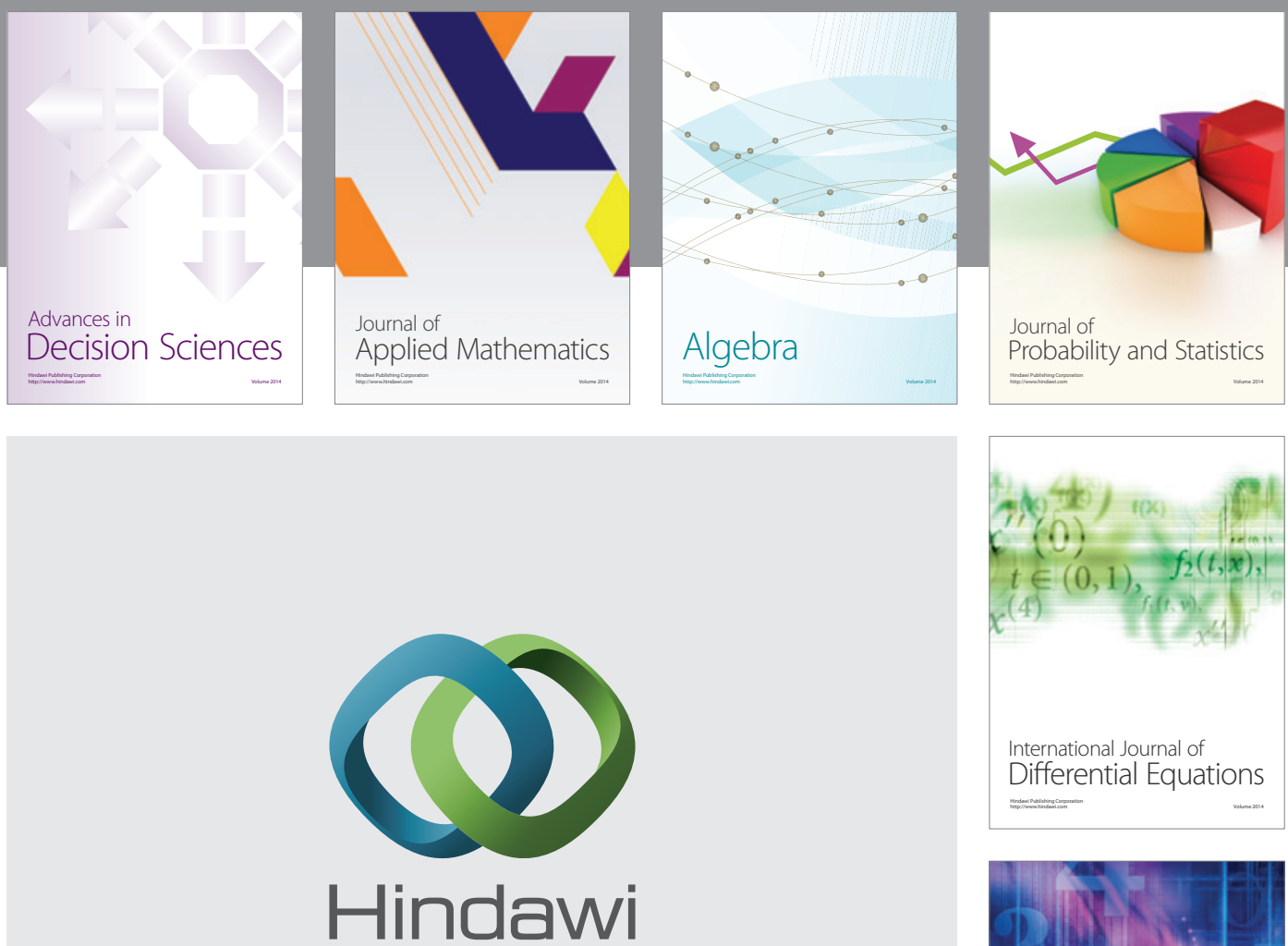

Submit your manuscripts at http://www.hindawi.com
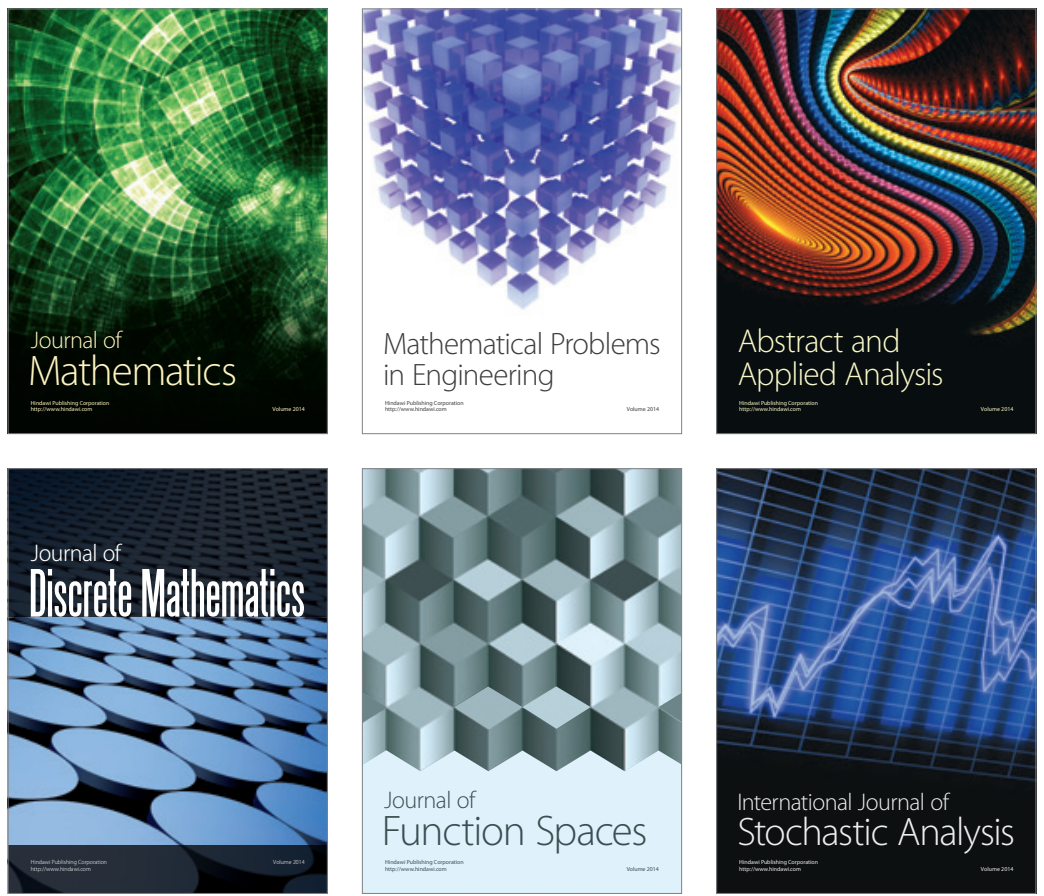

Journal of

Function Spaces

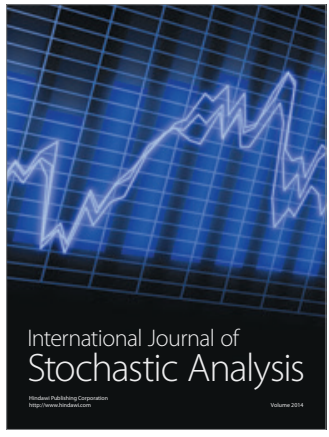

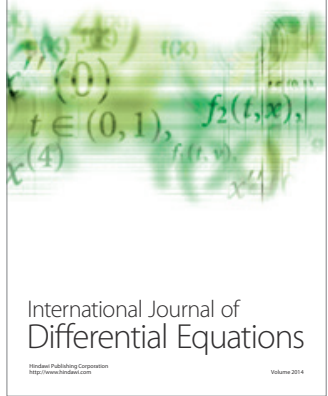
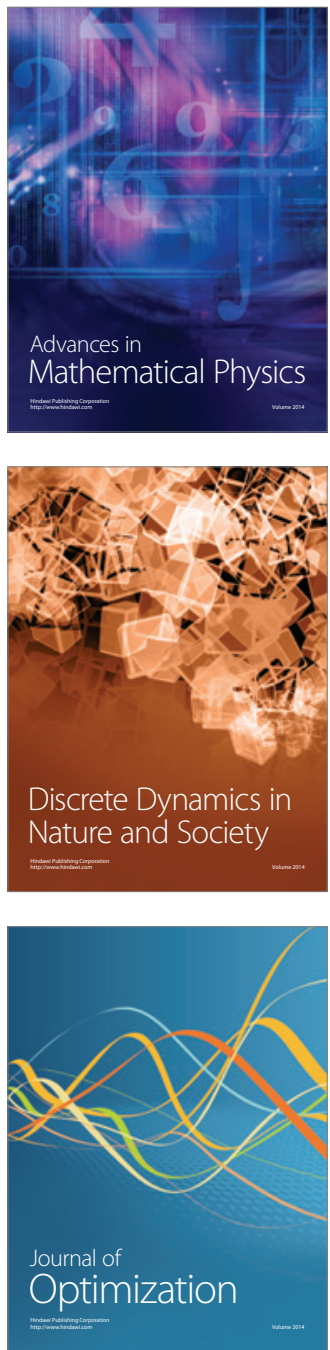